\title{
Assessing burn severity using satellite time series
}

\author{
S. Veraverbeke ${ }^{1}$, S. Lhermitte $^{2}$, W. Verstraeten ${ }^{3} \&$ R. Goossens ${ }^{1}$ \\ ${ }^{I}$ Department of Geography, Ghent University, Belgium \\ ${ }^{2}$ Centro de Estudios Avanzados en Zonas Aridas, \\ Universidad de la Serena, Chile \\ ${ }^{3}$ Department of Biosystems, Katholieke Universiteit Leuven, Belgium
}

\begin{abstract}
In this study a multi-temporal differenced Normalized Burn Ratio $\left(\mathrm{dNBR}_{\mathrm{MT}}\right)$ is presented to assess burn severity of the 2007 Peloponnese (Greece) wildfires. Eight-day composites were created using the daily near infrared (NIR) and mid infrared (MIR) reflectance products of the Moderate Resolution Imaging Spectroradiometer (MODIS). Prior to the calculation of the $\mathrm{dNBR}_{\mathrm{MT}}$, a pixel-based control plot selection procedure was initiated for each burned pixel based on time series similarity of the pre-fire year 2006 to estimate the spatio-temporal NBR dynamics in the case that no fire event would have occurred. The $\mathrm{dNBR}_{\mathrm{MT}}$ is defined as the one-year post-fire integrated difference between the NBR values of the control and focal pixels. As such, the $\mathrm{dNBR}_{\mathrm{MT}}$ accounts for both the direct fire impact and vegetation responses. The $\mathrm{dNBR}_{\mathrm{MT}}$, based on coarse resolution imagery with a high temporal frequency, has the potential to become either a valuable complement to fine resolution Landsat dNBR mapping or an imperative option for assessing burn severity at a continental to global scale.
\end{abstract}

Keywords: differenced normalized burn ratio, fire severity, burn severity, MODIS, multi-temporal, vegetation regeneration, remote sensing.

\section{Introduction}

Wildfires play an integral role in the ecological functioning of many ecosystems, as they partially or completely remove the vegetation layer and affect post-fire vegetation composition. The assessment of post-fire effects is a major challenge 
to understanding the potential degradation processes after a fire and to comprehend an ecosystem's post-fire resilience. To name these post-fire effects, the terms fire severity and burn severity are often interchangeably used [1], describing the amount of damage the physical, chemical and biological changes or the degree of alteration that fire causes to an ecosystem. Some authors, however, suggest a clear distinction between both terms by considering the fire disturbance continuum [2], which addresses three different temporal fire effects phases: before, during and after the fire. In this context, fire severity quantifies the short-term fire effects in the immediate post-fire environment, whereas burn severity quantifies both the short- and long-term impact as it includes response processes (e.g. resprouting, delayed mortality) $[3,4]$.

In remote sensing studies burn severity is traditionally estimated using fine resolution imagery [5]. A popular approach, partly because of its conceptual simplicity, can be found in ratioing band reflectance data. Both the Normalized Difference Vegetation Index (NDVI) and the Normalized Burn Ratio (NBR) are frequently used in this context; however, the latter has become accepted as the standard spectral index for assessing fire impact. The NBR relates to vegetation moisture content by combining the near infrared (NIR) and mid infrared (MIR) spectral regions, in which large post-fire reflectance changes occur. However, when using mono-temporal post-fire imagery, unburned sparsely vegetated areas and burned areas are often confounded [4]. Therefore pre- and post-fire NBR images are generally bi-temporally differenced, resulting in the differenced NBR (dNBR), which permits a clear contrast between burned and unburned regions.

The dNBR method relies on Landsat imagery and is thus dependent of fine resolution image availability, which is limited to infrequent images over small areas. As a result, fine resolution burn severity studies have proven to be valuable for obtaining detailed information over specific fires; however, they fail to provide a multi-temporal overview of burn severity on a regional to global scale. Moreover, the lag, i.e. the time since the fire, and seasonal timing of the Landsat bi-temporal dNBR assessment influence the burned pixels' dNBR variability and the absolute magnitude of change $[4,6]$. With regards to seasonal timing, an assessment during the green and productive stage of the vegetation contains a higher variability in post-fire effects than an assessment made in the dry season [4]. With respect to lag timing, Veraverbeke et al. [6] demonstrated that three weeks post-fire was the best time to estimate the fire impact with the dNBR, because then the fire-induced reflectance changes were maximal, which also resulted in more optimal pre/post-fire pixel displacements in the bi-spectral feature space. These temporal dissimilarities greatly limit the comparison between bi-temporal dNBR assessments of different fires, especially when a comparison between different ecoregions is required [5]. The use of multitemporal data could possibly allow this comparison, as they permit the integration of the temporal dimension in a more robust way. To date very few studies have implemented coarse resolution time series to assess burn severity. The aim of this study therefore is the introduction of a multi-temporal dNBR $\left(\mathrm{dNBR}_{\mathrm{MT}}\right)$ MODIS burn severity assessment approach based on minimum NIR 
NBR composites. The method accounts for both the direct fire impact and vegetation regeneration [3].

\section{Data}

The study area is situated in the Peloponnese peninsula, in southern Greece $\left(36^{\circ} 30^{\prime}-38^{\circ} 30^{\prime} \mathrm{N}, 21^{\circ}-23^{\circ} \mathrm{E}\right)$. After a severe drought period, several large wildfires of unknown cause struck the area in the summer of 2007. The fires consumed more than 150000 ha of coniferous forest, broadleaved forest, shrub lands (maquis and phrygana communities) and olive groves.

Level 2 daily Terra MODIS surface reflectance $(500 \mathrm{~m})$ tiles covering the study area (MOD09GA), including associated Quality Assurance (QA) layers, were acquired from the National Aeronautics and Space Administration (NASA) Warehouse Inventory Search Tool (WIST) (https://wist.echo.nasa.gov) for the period 01/01/2006-31/12/2008. The study area was clipped and the NIR (centered at $858 \mathrm{~nm}$ ), MIR (centered at $2130 \mathrm{~nm}$ ) and QA layers were extracted. Subsequently, the daily NIR, MIR and QA data were converted in eight-day composites using the minimum NIR criterion. After compositing a local secondorder polynomial function, also known as an adaptive Savitzky-Golay filter [7], was applied to the time series as implemented in the TIMESAT software [8] to replace bad QA observations. Finally, the NBR index was calculated as:

$$
N B R=\frac{N I R-M I R}{N I R+M I R}
$$

\section{Methodology}

Before applying the $\mathrm{dNBR}_{\mathrm{MT}}$ (see section 3.2) approach it was necessary to create a burned area map, to extract per pixel fire dates and to select control pixels that estimate the NBR's spatio-temporal behavior for the case that there would not have been a fire occurrence (see section 3.1).

\subsection{Preliminary processing}

The burned area and fire date were extracted based on the characteristic persistency of the post-fire NBR drop, similar to the algorithm of Chuvieco et al. [9]. The accuracy of the burned area map was verified using a burned area map derived from Landsat Thematic Mapper (TM) imagery [10]. MODIS burned area statistics were extracted windows of 10 by $10 \mathrm{~km}$. These statistics were regressed against their TM equivalents, in which the TM data served as independent variable and the MODIS data as dependent variable.

To minimize external and phenological variations a pixel based control plot selection method [11] was implemented. This control pixel selection is based on the similarity between the time series of the burned pixel and the time series of its surrounding unburned pixels for a pre-fire year. The selection is based on the 
time series similarity between pixels during the pre-fire year 2006 and the averaged Euclidian distance $D$ was used as dissimilarity measure:

$$
D=\frac{\sqrt{\sum_{t=1}^{N}\left(N B R_{t}^{f}-N B R_{t}^{x}\right)^{2}}}{N}
$$

where $N B R_{t}^{f}$ and $N B R_{t}^{x}$ are the respective burned focal and unburned candidate control pixel time series, while $\mathrm{N}$ is the number of observations in the pre-fire year $(N=46)$.

Application of the pixel based control plot selection method strongly depends on the size of the contextual neighborhood around the focal pixel, which determines the number of unburned control pixels $x$, and the number of selected control pixels $c$, since the combination of $x$ and $c$ will determine how well control pixels provide the temporal profile of the focal pixel as its best estimate in the case of no fire occurrence. For each burned focal pixel $f$ a user-defined number of unburned candidate pixels $x$ was selected. The candidate pixels are selected by increasing the spatial neighborhood window around $f$ with one pixel on each side till the user-defined threshold is met. Subsequently, a user-defined number of pixels $c$ that show the lowest temporal dissimilarity $D$ with $f$ is selected from the $x$ candidate pixels. Then, the dissimilarity $D$ was defined for the averaged time series of the $c$ pixels as the existence of a beneficial averaging effect that removes random noise in the time series has been perceived in previous research [11]. The dissimilarity $D$ is calculated for different settings of $x$ and $c$ to identify the most optimal control pixel selection conditions.

\subsection{Multi-temporal dNBR}

Burn severity incorporates both short-and long-term post-fire effects on the environment [3]. Consequently, burn severity is a combination of immediate fire impact and the ecosystem's ability to regenerate. Based on these characteristics, we propose a multi-temporal dNBR $\left(\mathrm{dNBR}_{\mathrm{MT}}\right)$ that integrates the difference between the NBR values of a burned pixel and its corresponding control pixel over time. Doing so the $\mathrm{dNBR}_{\mathrm{MT}}$ is defined as:

$$
d N B R_{M T}=\frac{\sum_{t=1}^{N}\left(N B R_{t}^{f}-N B R_{t}^{c}\right)}{N}
$$

where $N B R_{t}^{f}$ and $N B R_{t}^{c}$ are the respective burned focal and unburned control pixel observations, while $N$ is the number of post-fire observations included in the study (here $\mathrm{N}=46$ for one year) and $t=1$ is the first post-fire observation. Figure 1 illustrates the principle of the $\mathrm{dNBR}_{\mathrm{MT}}$. Dividing by the number of postfire observations $N$ normalizes the $\mathrm{dNBR}_{\mathrm{MT}}$ data to the same range as bitemporal dNBR assessments. 


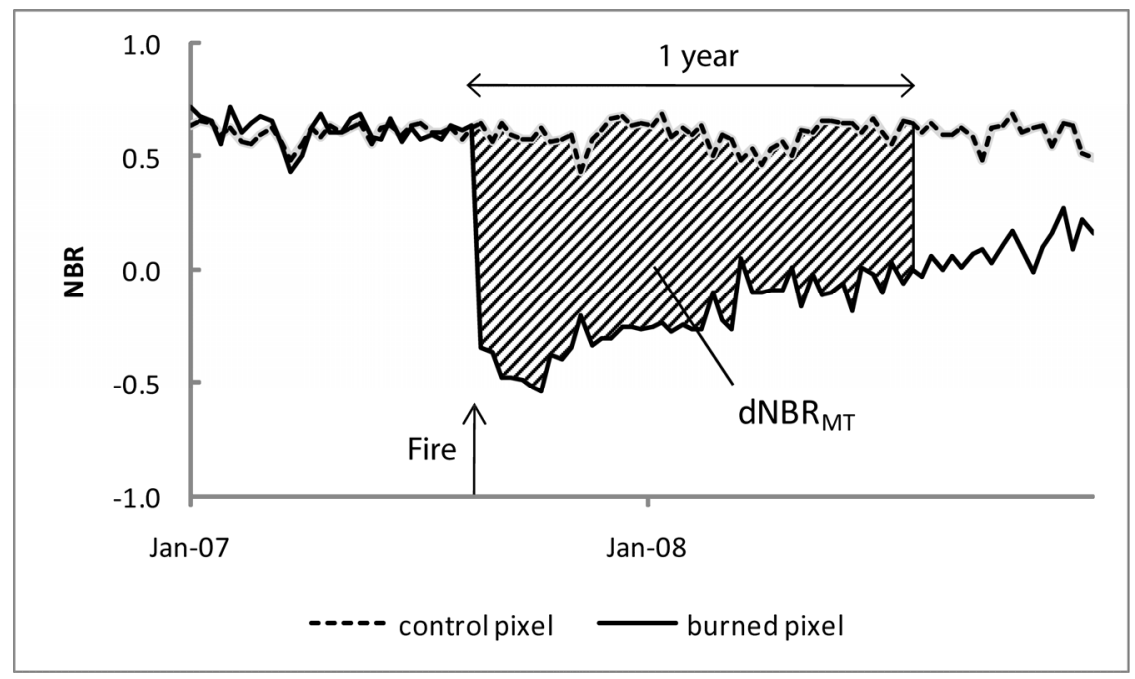

Figure 1: $\quad$ Principle of the multi-temporal dNBR ( $\left.\mathrm{dNBR}_{\mathrm{MT}}\right)$. The $\mathrm{dNBR}_{\mathrm{MT}}$ represents the averaged integrated difference between the one-year post fire NBR time series of the control and focal pixels, as shown in the figure by the shaded area.

\section{Results}

\subsection{Preliminary processing}

Figure 2 displays the distribution plot and regression line of the TM-MODIS comparison. The MODIS-derived burned area map correlated fairly well with the Landsat-based map (coefficient of determination $\mathrm{R}^{2}=0.98, \mathrm{p}<0.001$ ), although a consistent overestimation relative to the TM data was perceived as indicated by the regression slope of 1.31 .

Figure 3 demonstrates the differences in mean dissimilarity $D$ for different settings of the number of candidate and control pixels based on time series data of the pre-fire year 2006. Two main effects can be observed in the graph. Firstly, there was a consistently decreasing trend in dissimilarity $D$ when the number of unburned candidate pixels increased. This feature appeared regardless of the number of control pixels chosen. Secondly, the number of control pixels chosen also influenced the dissimilarity measure due to an averaging effect. The strength of this averaging effect was again dependent of the number of candidate pixels: the more candidate pixels, the more important the averaging effect. The averaged time series of the five most similar control pixels resulted in a mean dissimilarity $D$ of 0.0073 . The results are satisfying since the mean absolute difference between the NBR values of the control pixels and the focal pixels was lower than 0.05 (eqn 2). We used this setting to calculate NBR images of the years 2007 and 2008 , as best estimates of the spatio-temporal NBR dynamics in the case of no fire. 
112 Modelling, Monitoring and Management of Forest Fires II

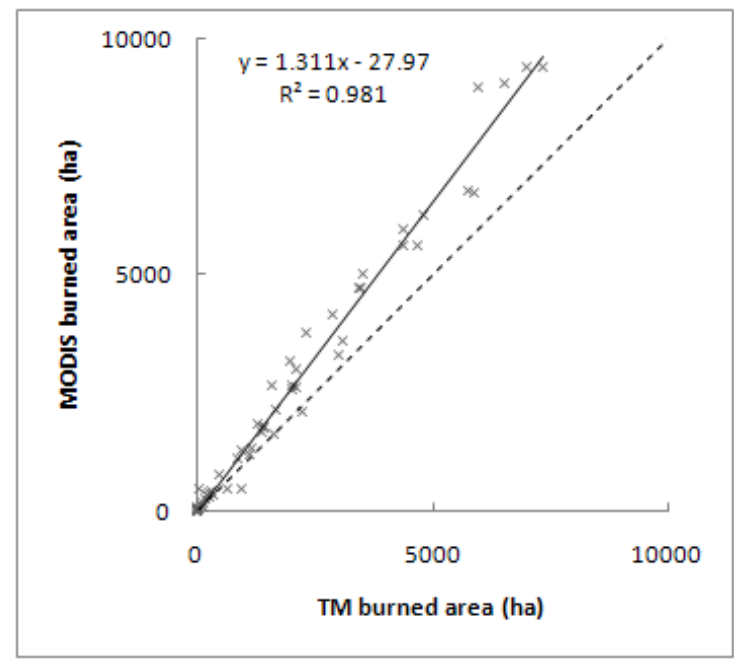

Figure 2: Distribution plot and regression line of the TM-MODIS burned area map comparison (regression significance of $\mathrm{p}<0.001$ ).

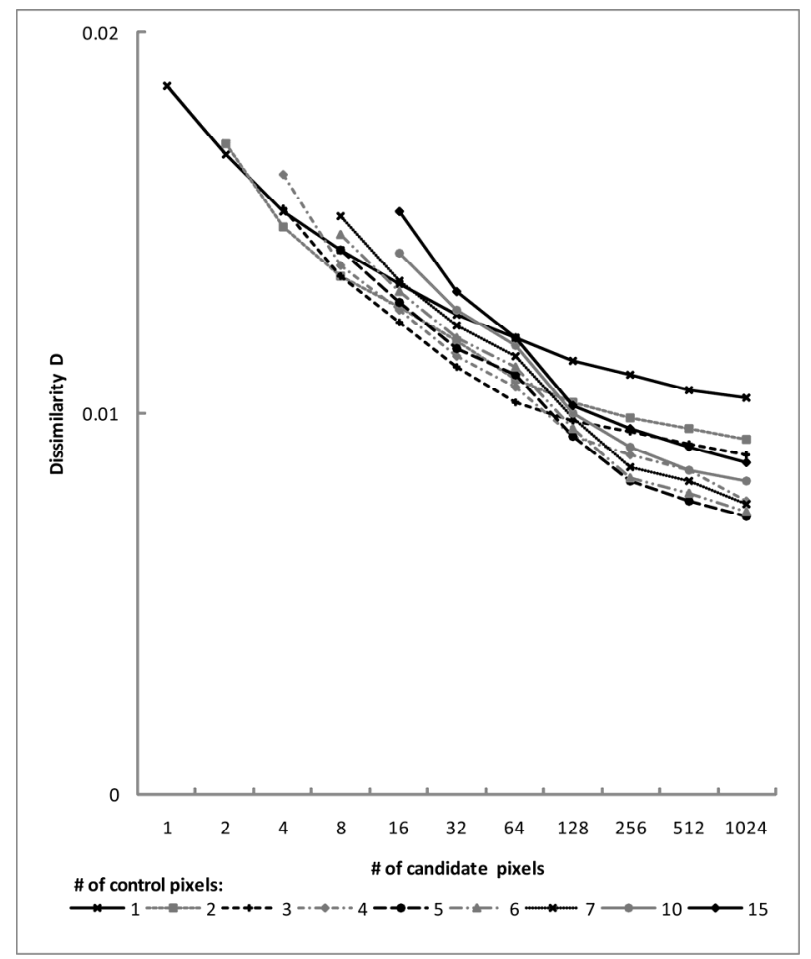

Figure 3: Mean dissimilarity $D$ in function of the number of candidate and control pixels calculated based on NBR time series data of the pre-fire year 2006. 


\section{$4.2 \mathrm{dNBR}_{\mathrm{MT}}$}

Figure 4A illustrates the result of the multi-temporal burn severity approach, while figure 5B details a specific burned area framed in blue in figure 4A. The images are mapped in a yellow-red color scale. Low $\mathrm{dNBR}_{\mathrm{MT}}$ values are represented in yellow, while a red color was assigned to high severity pixels. The spatial pattern of burn severity is clearly visible in the maps with an evident differentiation between high and low severity patches with relative smooth interpatches transitions. The mean $\mathrm{dNBR}_{\mathrm{MT}}$ equaled $0.27(\mathrm{sd}=0.14)($ figure $4 \mathrm{C})$.

Figure 5 shows the temporal profiles of a low, a moderate and a high severity pixel, A, B and C, respectively and their corresponding control pixels. These figures demonstrate that burn severity can be regarded as a combination of fire impact and the ability to recover. Although the three pixels experienced a clear NBR drop in the first post-fire weeks, the rate of recovery remarkably differed among the three pixels. Figure $5 \mathrm{~A}$ reveals a very fast regeneration after the initial NBR drop. Half a year after the fire, NBR values of the burned pixel almost approached the control pixel's values. In contrast, in the example of figure $5 \mathrm{C}$ the burned pixel illustrated a more gentle post-fire increase in NBR. In this respect, the example pixel of figure $5 \mathrm{~B}$ showed a somewhat intermediate behavior.
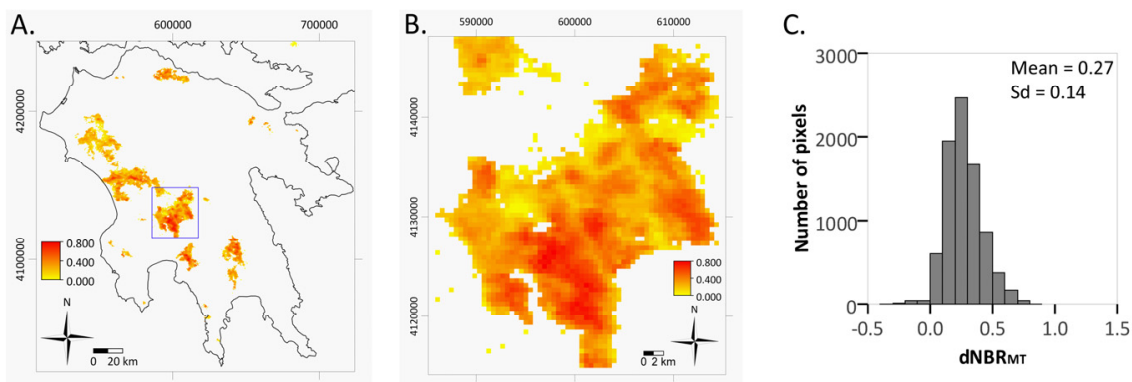

Figure 4: $\quad d_{N B R_{M T}}$ map (A), detailed subset $d_{N B R_{M T}}$ map of the rectangle in $A(B)$ and $\mathrm{dNBR}_{\mathrm{MT}}$ histogram (C).

\section{Discussion}

\subsection{Preliminary processing}

The regression model between TM and MODIS burned area statistics resulted in a high correlation $\left(\mathrm{R}^{2}=0.98\right)$ (see figure 3 ). Many authors have used fine resolution imagery to validate coarse resolution burned area maps (a.o. [12]). In some of these studies the coarse resolution approach produced an underestimation of the area burned relative to the fine resolution estimates (a.o. [12]), while in others an overestimation was perceived (a.o [12]). Although also related to vegetation type, the over- or underestimation can be explained by the burned area mapping algorithm's sensitivity to detect partially burned pixels 
[12]. If partially burned pixels are easily detected, this will result in an overestimation of the total burned area. In our study a clear overestimation was observed as indicated by the regression slope of 1.31. This overestimation is due to the relatively relaxed threshold applied in the bi-temporal differencing of NBR images. This minimized the omission error, at the expense of a higher commission error. The control pixel selection procedure, however, requires a total exclusion of burned pixels, even when they are only partially burned. A more precise threshold would favor the selection of partially burned pixels as potential control pixels. This would result in a potentially flawed simulation of a control pixel's temporal NBR development in the post-fire stage [13].
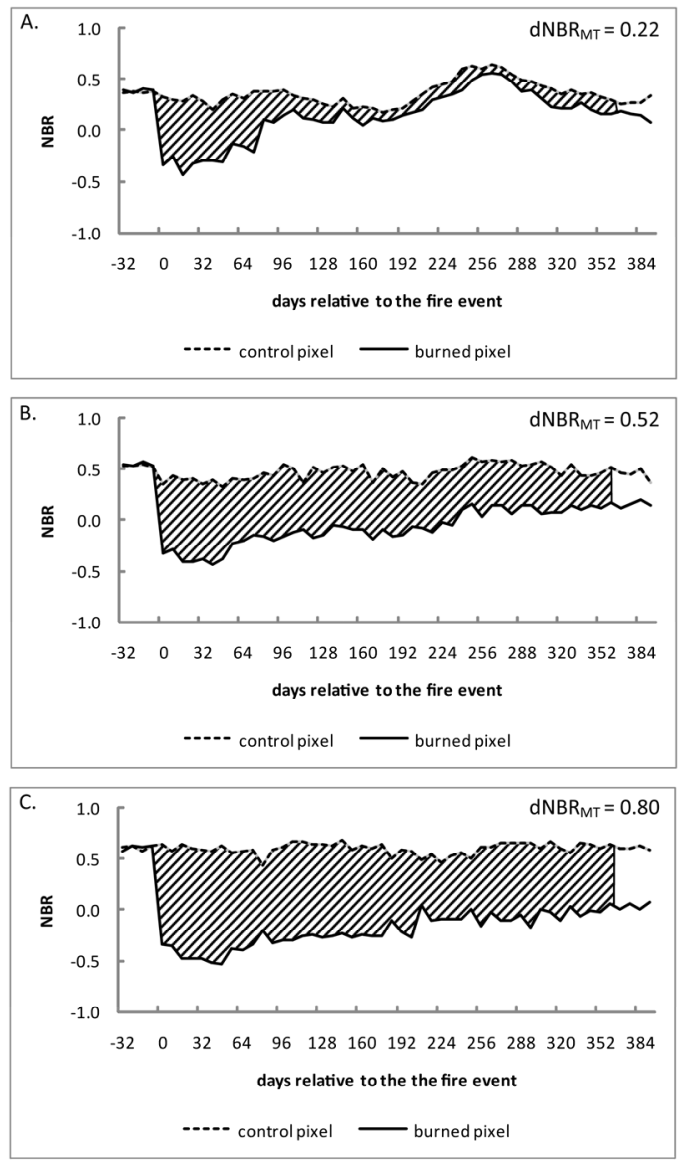

Figure 5: Illustration of low (A, 605600E 4131800N), moderate (B, $601400 \mathrm{E}, 4130400 \mathrm{~N})$ and high $(\mathrm{C}, 600900 \mathrm{E}, 4123500 \mathrm{~N})$ severity $\mathrm{dNBR}_{\mathrm{MT}}$ pixels. The area incorporated in the $\mathrm{dNBR}_{\mathrm{MT}}$ calculation is shaded. 
The pixel-based control plot selection using a one year pre-fire time series revealed two main effects. Firstly, mean dissimilarity $D$ decreased for an increasing number of candidate pixels, regardless of the number of control pixels chosen (see figure 3). This is trivial as by enlarging the spatial neighborhood around the focal burned pixel the probability of finding more similar pixels increases [11]. The relative decrease in dissimilarity is smaller when the number of candidate pixels doubles. This means that it is more likely that the most similar pixel is found close by the focal pixel than further away [11]. This corroborates with the recommendation of $\mathrm{Li}$ et al. [13] to select reference plots in the proximity of the burned areas. This is trivial as it follows a general rule that terrain features in a close vicinity are more likely to be similar than distant features. A second effect visible in figure 3 is the beneficial averaging effect, which became more obvious for a larger number of candidate pixels. This is rather remarkable as one would expect that the use of only the most similar pixel would give the best result. However, noise reduces the similarity between the most similar control and the focal pixel. Even after preprocessing, a certain amount of random noise remains present in the dataset. Averaging the two or more most similar pixels causes a more temporally stable signal because random noise is averaged out resulting in a higher temporal similarity with the focal pixel [11]. This beneficial averaging effect is, however, finite as at a certain point nonsimilar pixels will also be included in the averaging process, which will deteriorate the similarity. The number of pixels that must be included in the averaging process to reach the lowest dissimilarity also depends on the number of candidate pixels as a consequence of the higher probability to find more similar pixels among a larger amount of candidate pixels. So for a certain number of candidate pixels there exists a balance between favorable averaging and inclusion of dissimilar pixels. For our case the optimal balance was found by averaging the five most similar pixels out of 1024 control pixels.

\section{$5.2 \mathrm{dNBR}_{\mathrm{MT}}$}

A major advantage of the multi-temporal burn severity approach is its combination of both the immediate fire impact and vegetation regrowth. As such, it is more tightly connected to the definition of burn severity. Key [4] stated that burn severity encloses both first and second order fire effects. The most important first order effect is the fire's vegetation consumption, while vegetation regeneration and delayed mortality are substantial second order effects. In that respect, Lentile et al. [3] specified that burn severity relates to the amount of time necessary to return to pre-fire level. As such, they made a clear distinction between burn and fire severity, where the latter term is restricted to the immediate fire impact only. Bi-temporal assessments can only partly contribute to a thorough interpretation of burn severity as they fail to include post-fire vegetation responses. As seen in figure 5 the manifestation of regeneration processes can be diverse. Furthermore the degree of ecological information in bitemporal studies is highly dependent on the timing of the assessment $[4,6]$. Thus, where fine resolution Landsat studies allow revealing a high spatial detail, they miss the comprehension of the temporal dimension of burn severity. 
Therefore, the higher temporal frequency of coarse resolution imagery can either be a vital complement to traditional Landsat dNBR mapping of specific fires or an imperative alternative for the assessment of burn severity at continental to global scales. Another strength of the $\mathrm{dNBR}_{\mathrm{MT}}$ is its insensitivity to inter-annual phenological differences, which frequently distort traditional pre/post-fire detection schemes [14]. This characteristic is due to the pixel-based control plot selection procedure [11]. Comparing the condition of a burned pixel with how the pixel would have behaved in the case of no fire, as estimated by the selected control plot within the same image, largely reduces external influences.

One of the prime concerns of scientists involved with burn severity mapping is the categorization of the fire-affected pixels in severity classes [4]. This duty is, however, to some part problematic due to the difficulty to determine absolute threshold that are ecologically meaningful across several ecoregions [15]. A first attempt to ameliorate the comparison of burn severity in different fires was made by Miller and Thode [15], who proposed a relative version of the dNBR (RdNBR). This index assesses the degree of environmental change caused by the fire relative to the pre-fire conditions. Therefore, more than the absolute index, the RdNBR hypothetically allows a better comparison among different land cover types, especially in heterogeneous landscapes. The improvement was made clear for fires in conifer dominated vegetation types in California, USA [16], although in other studies the correlation between the RdNBR and field estimates of severity was weaker than observed with the dNBR [17]. Miller and Thode [15] explicitly state that the implementation of a relative index will allow a more direct comparison of severity between fires across space and time. Whether the hypothetical advantage of the relative index to account for spatial heterogeneity has an intuitive appeal, the index does not handle temporal differences which may be present among different assessments. This is due to two main effects. Firstly, vegetation recovery decreases the vigor of the fire-induced change with time [6, 17], especially in quickly recovering ecotypes such as in the Mediterranean. Secondly, the seasonal timing determines the vegetation productivity and wetness of both the control and burned plots which influences the absolute magnitude of change and the degree of variability in the dNBR data $[4,6]$. These two effects potentially hamper any comparison of two bi-temporal dNBR assessments and the issue has also a close linkage with the recent confusion in post-fire effects terminology (fire severity, burn severity, ecosystem response, etc.) [1]. Therefore caution is advised when using the dNBR to monitor and compare trends in burn severity either in time or across regions. The $\mathrm{dNBR}_{\mathrm{MT}}$, however, integrates the temporal variability into one value. If the period to which the integration is applied remains the same for different fires, the multi-temporal approach truly has the potential to allow a better comparison of burn severity either in time or space.

\section{Conclusions}

MODIS daily MIR and NIR reflectance products were used in a multi-temporal burn severity study of the 2007 Peloponnese (Greece) wildfires. Images were 
first composited in eight-day periods and subsequently missing values were replaced by means of the adaptive Savitzky-Golay filter. Prior to the calculation of the $\mathrm{dNBR}_{\mathrm{MT}}$ the burned area was mapped using the post-fire NBR drop characteristic. Then, for each burned pixel, a control plot was selected based on time series similarity of the pre-fire year 2006. Thanks to the control plot selection procedure the multi-temporal burn severity approach remained unaffected by phenological differences. After this preliminary processing, the $\mathrm{dNBR}_{\mathrm{MT}}$ was calculated as the one-year post-fire integrated difference between the NBR of the control and focal pixels, averaged by the total number of observations. By using a one-year post-fire time period, the $\mathrm{dNBR}_{\mathrm{MT}}$ incorporates both the immediate fire impact and the regeneration processes. These regeneration processes, combined with seasonal effects, make any bitemporal analysis dependent on the timing of the assessment and as a consequence the inter-comparability of dNBR assessments conducted in different fires suffers from this. The $\mathrm{dNBR}_{\mathrm{MT}}$, on the other hand integrates the one-year temporal variability into one value. The $\mathrm{dNBR}_{\mathrm{MT}}$, which is based on coarse resolution imagery with a high temporal frequency, has the potential to be either a valuable complement to fine resolution Landsat dNBR assessments or an imperative option for burn severity mapping at a continental to global scale with an enhanced comparability of different fires across space and time.

\section{References}

[1] Keeley, J., Fire intensity, fire severity and burn severity: a brief review and suggested usage. International Journal of Wildland Fire, 18, pp. 116-126, 2009.

[2] Jain, T., Pilliod, D., Graham, R., Tongue-tied. Wildfire, 4, pp. 22-26, 2004.

[3] Lentile, L., Holden, Z., Smith, A., Falkowski, M., Hudak, A., Morgan, P., Lewis, S., Gessler, P., Benson, N., Remote sensing techniques to assess active fire characteristics and post-fire effects. International Journal of Wildland Fire, 15, pp. 319-345, 2006.

[4] Key, C., Ecological and sampling constraints on defining landscape fire severity. Fire Ecology, 2, pp. 34-59, 2006.

[5] French, N., Kasischke, E., Hall, R., Murphy, K., Verbyla, D., Hoy, E., Allen, J., Using Landsat data to assess fire and burn severity in the North American boreal forest region: an overview and summary of results. International Journal of Wildland Fire, 17, pp. 443-462, 2008.

[6] Veraverbeke, S., Lhermitte, S., Verstraeten, W.W., Goossens, R., Assessment of the temporal sensitivity of the differenced Normalized Burn Ratio (dNBR) to estimate burn severity. Remote Sensing of Environment, in review, 2010.

[7] Savitzky, A., Golay, M., Smoothing and differentiation of data by simplified least squares procedures. Analytical Chemistry, 36, pp. 16271639, 1964.

[8] Jonsson, P., Eklundh, L., TIMESAT-a program for analyzing time-series of satellite sensor data. Computers and Geosciences, 30, pp. 833-845, 2004. 
[9] Chuvieco, E., Englefield, P., Trishchenko, A., Luo, Y., Generation of long time series of burn area maps of the boreal forest from NOAA-AVHRR composite data. Remote Sensing of Environment, 112, pp. 2381-2396, 2008.

[10] Veraverbeke, S., Verstraeten, W.W., Lhermitte, S., Goossens, R., Illumination effects on the differenced Normalized Burn Ratio's optimality for assessing fire severity. International Journal of Applied Earth Observation and Geoinformation, 12, pp. 60-70, 2010.

[11] Lhermitte, S., Verbesselt, J., Verstraeten, W.W., Coppin, P., A pixel based regeneration index using time series similarity and spatial context. Photogrammetric Engineering and Remote Sensing, in press, 2010.

[12] Silva, J., Sa, A., Pereira, J., Comparison of burned area estimates derived from SPOT-VEGETATION and Landsat ETM+ data in Africa: influence of spatial pattern and vegetation type. Remote Sensing of Environment, 96, pp. 188-201, 2005.

[13] Li, M., Qu, J., Hao, X., Detecting vegetation change with satellite remote sensing over 2007 Georgia wildfire regions. Journal of Applied Remote Sensing, 2, pp. 021505, 2008

[14] Verbyla, D., Kasischke, E., Hoy, E., Seasonal and topographic effects on estimating fire severity from Landsat TM/ETM+ data. International Journal of Wildland Fire, 17, pp. 527-534, 2008.

[15] Miller, J., Thode, A., Quantifying burn severity in a heterogenous landscape with a relative version of the delta Normalized Burn Ratio (dNBR). Remote Sensing of Environment, 109, pp. 66-80, 2007.

[16] Miller, J., Knapp, E., Key, C., Skinner, C., Isbell, C., Creasy, R., Sherlock, J., Calibration and validation of the relative differenced Normalized Burn Ratio (RdNBR) to three measures of fire severity in the Sierra Nevada and Klamath Mountains, California, USA. Remote Sensing of Environment, 113, pp. 645-656, 2009

[17] Allen, J., Sorbel, B., Assessing the differenced Normalized Burn Ratio's ability to map burn severity in the boreal forest and tundra ecosystems of Alaska's national parks. International Journal of Wildland Fire, 17, pp. 463-475, 2008.

[18] Veraverbeke, S., Lhermitte, S., Verstraeten, W., Goossens, R., Evaluation of pre/post-fire differenced spectral indices for assessing burn severity in a Mediterranean environment with Landsat Thematic Mapper. International Journal of Remote Sensing, in press. 